\title{
Interleukin-8 and Matrix Metalloprotease 9 as Salivary Biomarkers of Pain in Patients with Temporomandibular Disorder Myalgia: A Pilot Study
}

\author{
Yang Mi Park', Yong-Woo Ahn ${ }^{1,2}$, Sung-Hee Jeong ${ }^{1,2}$, Hye-Min Ju', \\ Hye-Mi Jeon ${ }^{3}$, Kyung-Hee Kim ${ }^{4}$, Soo-Min Ok ${ }^{1,2}$
}

Received November 25, 2019 Revised December 20, 2019 Accepted December 20, 2019
Purpose: To search the salivary factors that objectively indicate an pain in myalgia patients with temporomandibular joint disorder (TMD) and determine the possibility of the factors as pain-biomarkers.

Methods: Participants consisted of pain-free 15 persons (male 7, female 8, mean age \pm standard deviation (SD); $26.8 \pm 16.04$ years) and 45 myalgia patients with TMD (male 21 , female 24 , mean age $\pm S D ; 27.98 \pm 13.01$ years). They were divided into a pain-free group (numerical rating scale [NRS] score 0), a mild pain group (NRS 1-4), a moderate pain group (NRS 5-6), and a severe pain group (NRS 7-10) and members of all groups were age, sex matched. Interleukin-8 (IL-8) and matrix metalloprotease 9 (MMP-9) were selected as pain biomarkers, by searching the Gene Expression Omnibus database and analyzing pain-related genes. Enzyme-linked immunosorbent assays were used to measure the concentration of IL-8 and MMP-9 in the patients' saliva.

Results: IL-8 and MMP-9 levels were statistically significantly higher in pain groups than in the pain-free group. Greater differences were observed in patients with acute pain (with painful duration under 3 months) than in the control group and in female patients than in male.

Conclusions: Salivary IL-8 and MMP-9 may play a role as biomarkers of myalgia in patients with TMD.

Key Words: Biomarkers; Interleukin-8; Matrix metalloprotease 9; Myalgia; Saliva; Temporomandibular joint disorders

\section{INTRODUCTION}

Pain is the main cause of patient visits to a clinic and one of the major symptoms experienced by patients attending a clinic [1]. However, pain is completely subjective, and thus clinicians have no choice but to assume the level of the patient's pain through their statements, gestures, and facial expressions. A description of the pain level is impossible for the mental retarded, infants, and people with a speech disorder [2,3]. Therefore, clinicians are limited in their evaluation of the severity, modality, and changes in a patient's pain, and may overestimate or underestimate their pain, and cannot provide them with the appropriate medical care.

The nature of pain is generally evaluated in terms of intensity [4]. To date, a visual analogue scale (VAS), verbal rating scale (VRS), and numerical rating scale (NRS) have

Copyright (c) 2019 Korean Academy of Orofacial Pain and Oral Medicine. All rights reserved.

(c) This is an open-access article distributed under the terms of the Creative Commons Attribution Non-Commercial License (http://creativecommons.org/licenses/by-nc/4.0/), which permits unrestricted non-commercial use, distribution, and reproduction in any medium, provided the original work is properly cited. 
been used to measure pain intensity in a clinic context and in academic research $[5,6]$. Patients convey their pain to the clinician by means of a VAS, VRS, or NRS, using a picture, language, or number, respectively, to indicate no pain to the worst possible pain. However, the answers are dependent on the instructions of how to mark the pain rating scales and patients with communication barriers cannot use these methods. Additionally, the three rating scales also are influenced by the patient's subjectivity and bias $[5,6]$. Hence, objective pain-measuring methods are needed to make an accurate diagnosis of pain, apply an appropriate remedy, evaluate a prognosis and develop effective analgesics.

Muscle pain is the most common complaint of patients with painful temporomandibular disorder (TMD) [7]. As many as 50 to 70\% of all TMD patients report masticatory muscle pain, and this is the second most common musculoskeletal condition (after chronic low back pain) that results in pain and disability $[7,8]$. Patients with TMD myalgia have incomplete mouth opening, and are restricted in their ability to talk and eat because of this limited mandibular movement; the condition is further influenced by psychosocial stress [8]. Therefore, correct evaluation of myalgia in TMD patients is necessary, not only to cure the disease, but also to improve the patient's quality of life.

Salivary testing has many advantages as a diagnostic tool; it is non-invasive, easy to use, inexpensive, and allows real-time diagnosis $[9,10]$. Thus, saliva has been extensively studied to search for biomarkers of various cancers and acute myocardial infarction, such as cancer antigen 125 (CA125), C-reactive protein, myoglobin, and myeloperoxidase $[11,12]$. Objective measurements of pain could also be effectively used for making an accurate diagnosis, assessing treatment results, managing patients who are more vulnerable to pain, and determining underlying pain mechanisms [13].

The goals of this study were to find salivary pain-detecting candidates of myalgia in patients with TMD, to compare the factors in the pain groups and in the pain-free group, and to evaluate the usefulness of the selected biomarkers.

\section{MATERIALS AND METHODS}

\section{Subjects}

This study was performed with 387 TMD patients who visited the Department of Oral Medicine, Pusan National University Dental Hospital in 2015. All patients provided informed consent for this study, and were examined for TMD. Exclusion criteria were patients taking any medication, patients with diabetes mellitus, hypertension, cardiovascular disease, and individuals with other painful diseases (e.g., odontalgia) or inflammatory diseases (e.g. oral inflammation). NRS were used to measure the patients' pain level. Finally, pain groups were composed of 45 TMD patients (male 21, female 24, mean age \pm standard deviation (SD); 27.98 \pm 13.01 years) with myalgia, diagnosed using the diagnostic criteria for TMD axis I, while the pain-free group were 15 individuals (male 7 , female 8 , mean age $\pm S D$; $26.8 \pm 16.04$ years) without pain (normal men, who had been evaluated for orthodontic treatment or who had diagnosed normal range). Subjects were divided into a pain-free group (NRS 0), a mild pain group (NRS 1-4), a moderate pain group (NRS 5-6), and a severe pain group (NRS 7-10). The groups each comprised of 15 gender- and age-matched patients (Fig. 1). The study protocol was approved by the Institutional Review Board of Pusan National University Dental Hospital (IRB no. \#PNUDH-2015-019).

\section{The Selection of Pain Markers}

To find pain biomarkers, the gene ontology database was searched using the following key words: pain, saliva, human and Homo sapiens. The Gene Expression Omnibus (GEO) database contains microarray data showing genes that are highly expressed in patients with stress, sleep deprivation, lung cancer, fibromyalgia, bladder pain syndrome, and complex regional pain syndrome (CRPS). Among them, CRPS is a chronic, progressive, and devastating pain syndrome and is an appropriate condition to use for searching for pain-related genes (http://www.ncb.nlm.nih.gov/ geo/; series GSE47603). It has been reported that there are 80 genes that are statistically significantly differentially expressed in CRPS patients. To find genes that are up-regulated according to pain severity, 69 genes were chosen as candidates [14]. Among these genes, matrix metalloprotease 


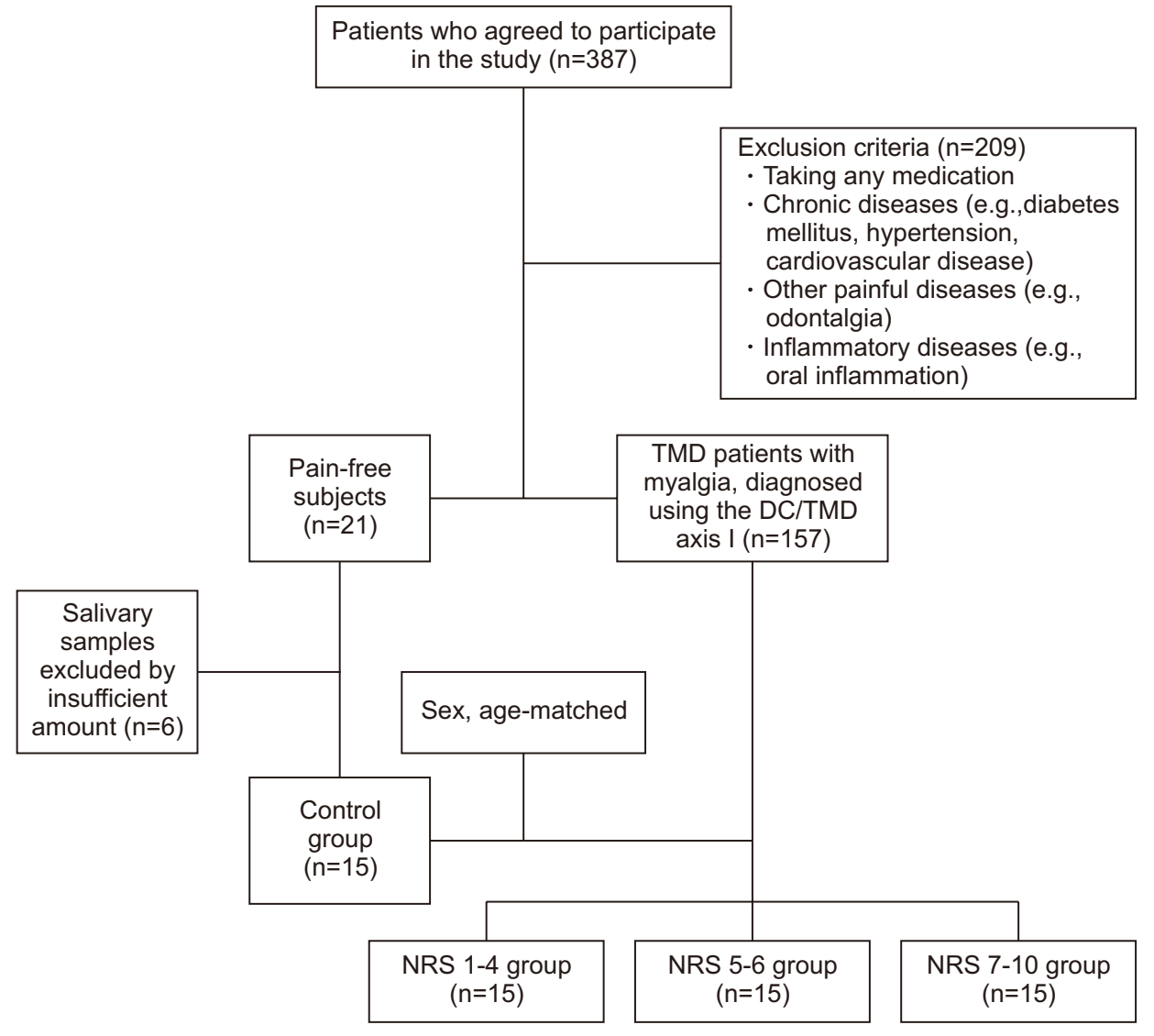

Fig. 1. Flow chart of this study population. TMD, temporomandibular joint disorder; DC/TMD, diagnostic driteria for TMD; NRS, numerical rating scale.
9 (MMP-9), prostaglandin endoperoxide synthase 2, interleukin-8 (IL-8), L-histidine decarboxylase, B cell translocation gene family, member 2 overlapped with genes known to be related to pain-recognizing systems. Finally, IL-8 and MMP-9 were selected as pain biomarkers, because they could be detected in saliva (Fig. 2).

\section{Collection of Saliva Samples}

After obtaining patient's informed consent, participants had oral examination and confirmed no gingival diseases or other inflammation. Saliva samples were collected from all patients at their first dental visit. During the collection, subjects were asked to slightly tilt their head forward and not to speak or consciously accumulate their saliva. All their unstimulated saliva was collected in a sterile polypropylene tube, over a period of 5 minutes. The saliva samples were immediately stored at $-80^{\circ} \mathrm{C}$ until assayed.

\section{Measurements of Salivary IL-8 and MMP-9}

Saliva samples were centrifuged at $13,000 \times g, 4^{\circ} \mathrm{C}$, for

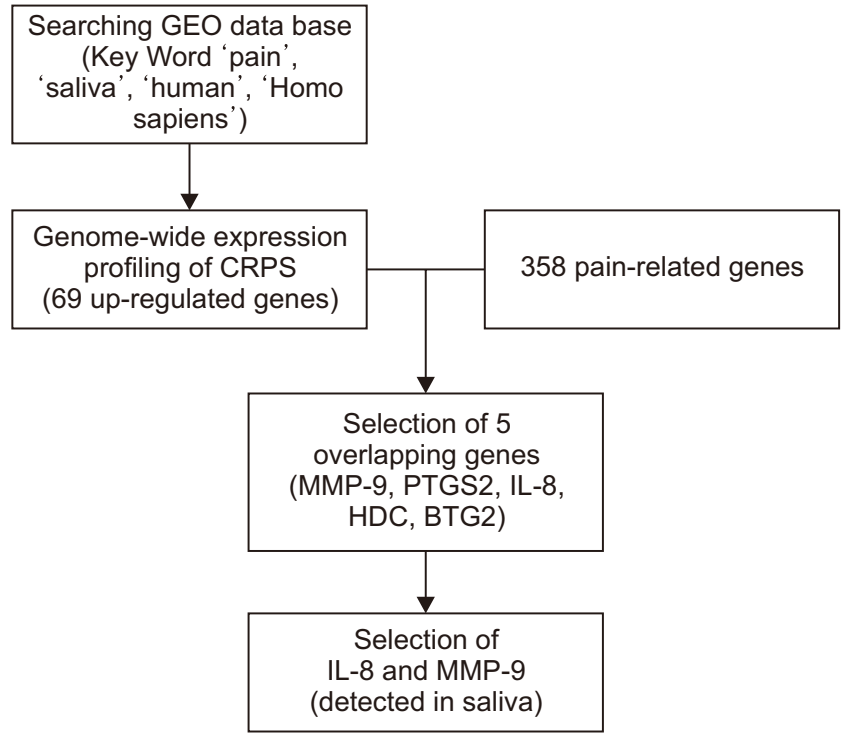

Fig. 2. Flow chart of selection process for salivary pain biomarkers. GEO, Gene Expression Omnibus; CRPS, complex regional pain syndrome; MMP-9, matrix metalloprotease 9; PTGS2, prostaglandin endoperoxide synthase $2 ; \mathrm{IL}-8$, interleukin-8; HDC, histidine decarboxylase; BTG2, B cell translocation gene family, member 2 . 
5 minutes. The levels of IL-8 and MMP-9 were measured using enzyme-linked immunosorbent assay kits according to the manufacturer's protocol (Duoset; R\&D Systems, Minneapolis, MN, USA). Standard curves were generated by using standards provided with the kits and the concentrations of salivary IL-8 and MMP-9 were calculated. All samples were tested in duplicate. Absorbance values were determined ( $\lambda=450 \mathrm{~nm}$ and $570 \mathrm{~nm}$ ) using a spectrophotometer.

\section{Comparison between IL-8 and MMP-9 Levels}

The levels of IL-8 and MMP-9 in the saliva were investigated according to the presence or absence of pain, intensity of pain, duration of myalgia and gender of subjects. In case of duration of pain, since a period of 3 months is widely used as dividing standard of acute and chronic pain [15], IL-8 and MMP-9 levels were analyzed in pain-free group, in the pain group suffering from pain for 3 months and in the pain group suffering from pain for more than 3 months.

\section{Statistical Analysis}

Statistical analyses were performed using IBM SPSS Statistics for Windows, Version 23.0 (IBM Co., Armonk, NY, USA). The Kruskal-Wallis test and analysis of variance were used to find meaningful differences between the groups. The Mann-Whitney U-test was used to determine the significance of differences between the pain groups and the control group. A p-values less than 0.05 were considered to indicate statistically significant differences.

\section{RESULTS}

\section{The relationship of IL-8 and MMP-9 Levels with Masticatory Muscle Pain}

The concentrations of salivary IL-8 were statistically higher in the pain groups (NRS 1-10) than in the pain-free group (NRS 0) $(\mathrm{p}<0.05)$ (Fig. 3). The IL-8 levels were more than twice as high in the mild pain group (NRS 1-4) than in the control group (NRS 0) and tended to decrease from the moderate pain group (NRS 5-6) to the severe pain group (NRS 7-10) $(\mathrm{p}<0.05)$ (Fig. 4).

The concentrations of salivary MMP-9 were also statistically higher in the pain groups (NRS 1-10) than in the painfree group (NRS 0) $(\mathrm{p}<0.05)$ (Fig. 3). The MMP-9 levels were about two-fold higher in the mild pain group (NRS 1-4) than in the control group (NRS 0) and tended to decrease from the moderate pain group (NRS 5-6) to the severe pain group (NRS 7-10) $(\mathrm{p}<0.05)$ (Fig. 4).

\section{The Relationship of IL-8 and MMP-9 levels with Duration of Pain}

Patients were divided into three groups: the pain-free group, the pain group suffering from for 3 months or less, and the pain group suffering from for more than 3 months. Compared to the pain-free group, the salivary concentrations of IL-8 and MMP-9 were significantly higher in the
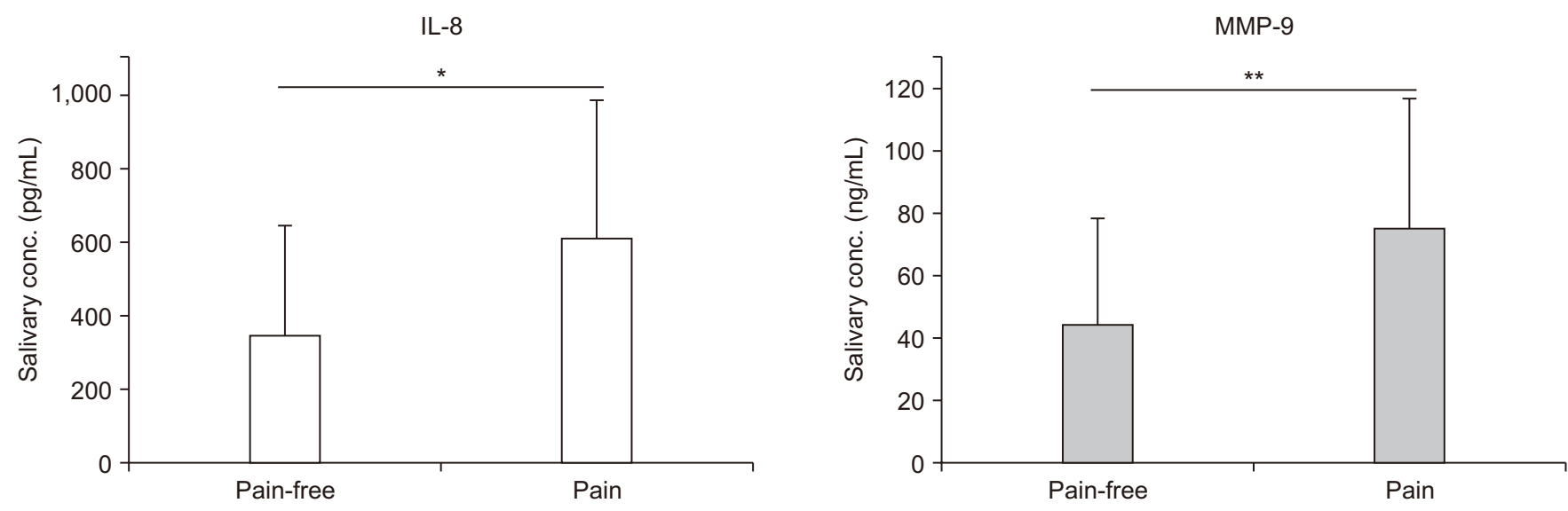

Fig. 3. Mean concentrations of IL-8 and MMP-9 in pain-free and pain groups. The concentrations of salivary IL-8 and MMP-9 were statistically higher in the pain groups than in the pain-free group $\left({ }^{*} * *_{p}<0.05\right)$. The Mann - Whitney U-test was performed to compare concentrations of IL-8 and MMP-9 between groups. IL-8, interleukin-8; MMP-9, matrix metalloprotease 9 
IL-8

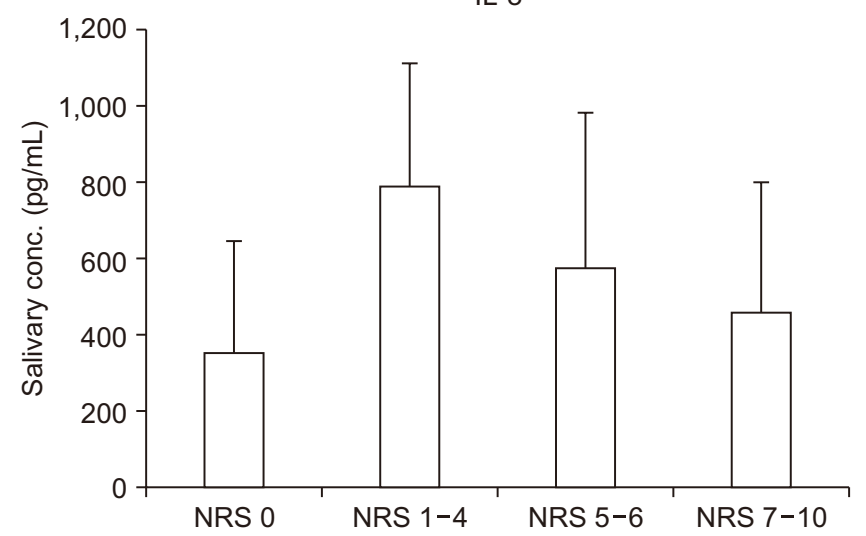

MMP-9

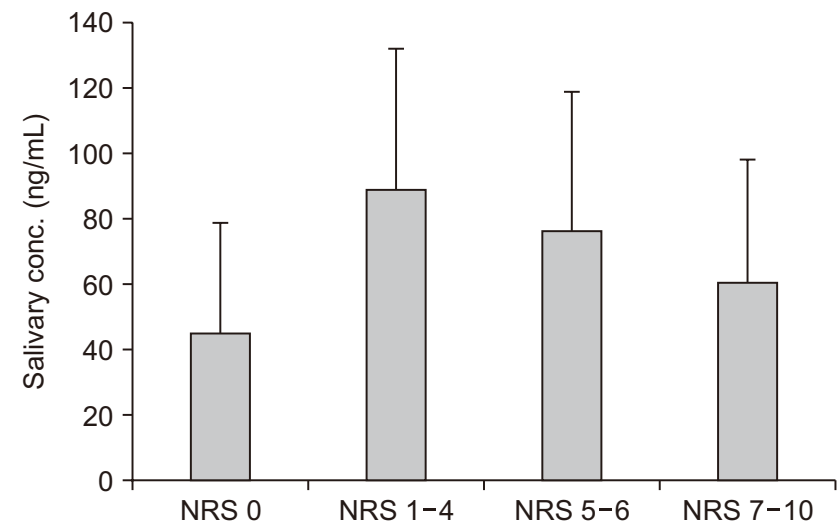

Fig. 4. Comparisons of mean concentrations of IL-8 and MMP-9 according to pain intensity. The IL-8 and MMP-9 levels were more than twofold higher in the mild pain group (NRS 1-4) than in the control group (NRS 0) and tended to decrease from the moderate pain group (NRS $5-6)$ to the severe pain group (NRS 7-10) $(p<0.05)$. The Kruskal-Wallis test was performed to compare the concentrations of IL-8 and MMP-9 between groups. IL-8, interleukin-8; MMP-9, matrix metalloprotease 9; NRS, numerical rating scale.

IL-8

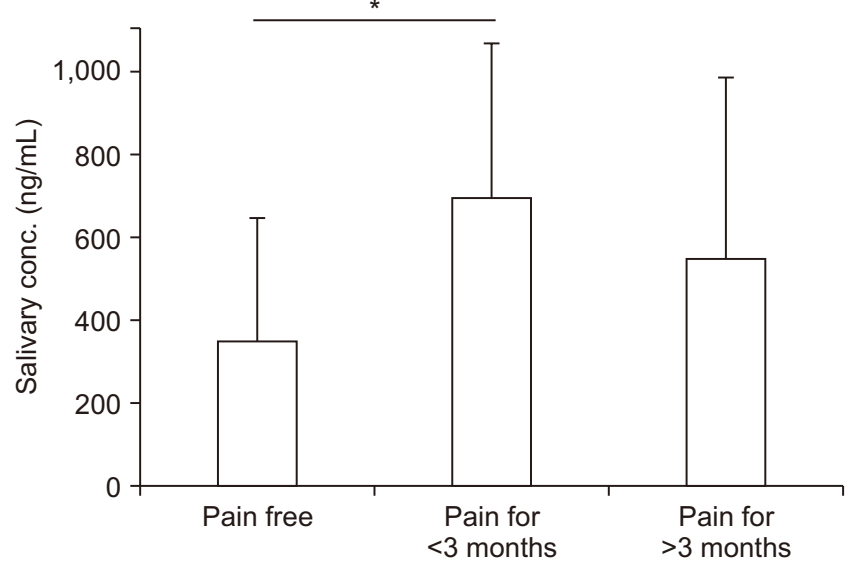

MMP-9

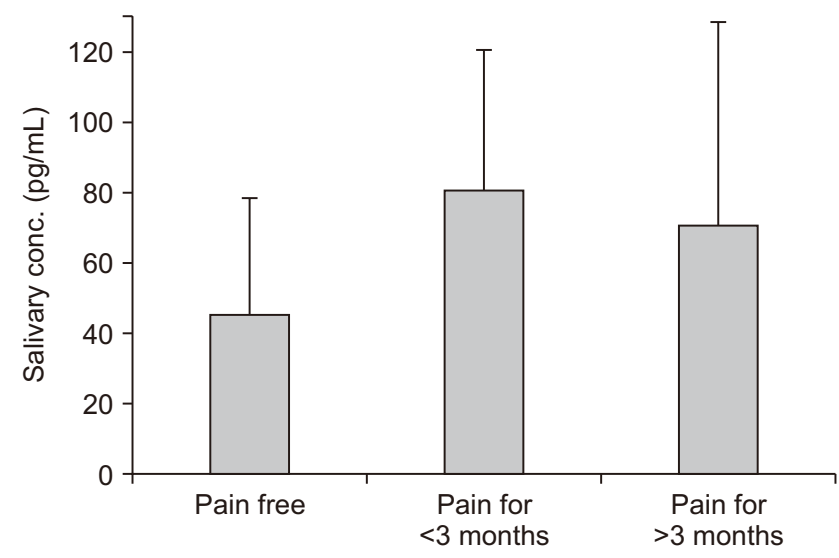

Fig. 5. Mean concentrations of IL-8 and MMP-9 in pain-free and pain groups according to the duration of pain. The concentrations of salivary IL-8 and MMP-9 were statistically higher in the pain groups suffering from pain for 3 months or less than in the pain-free group (**** $p<0.05$ ). The Mann - Whitney U-test was performed to compare concentrations of IL-8 and MMP-9 between groups. IL-8, interleukin-8; MMP-9, matrix metalloprotease 9 .

pain group suffering from for 3 months or less $(p<0.05)$ (Fig. 5). There was no meaningful relationship between pain severity and pain duration (data not shown).

\section{The Relationship of IL-8 and MMP-9 Levels with Sex}

The levels of IL-8 and MMP-9 were markedly higher in female pain group than in the pain-free group $(p<0.05)$ (Fig. 6). There was no meaningful difference between the painfree and pain group according to gender (data not shown).

\section{DISCUSSION}

To identify salivary biomarkers that could objectively indicate a TMD patient's pain, the GEO database and painrelated genes were analyzed. Consequently, IL-8 and MMP9 were selected as candidate biomarkers and analyzed the relation with myalgia.

IL-8 is a cytokine that functions as a mediator in the inflammatory response and is produced by blood cells, endothelial cells, and fibroblasts [16]. Increased levels of IL-8 have been reported in patients with chronic back pain $[17,18]$, fibromyalgia $[19,20]$, chronic fatigue syndrome [21] 
IL-8

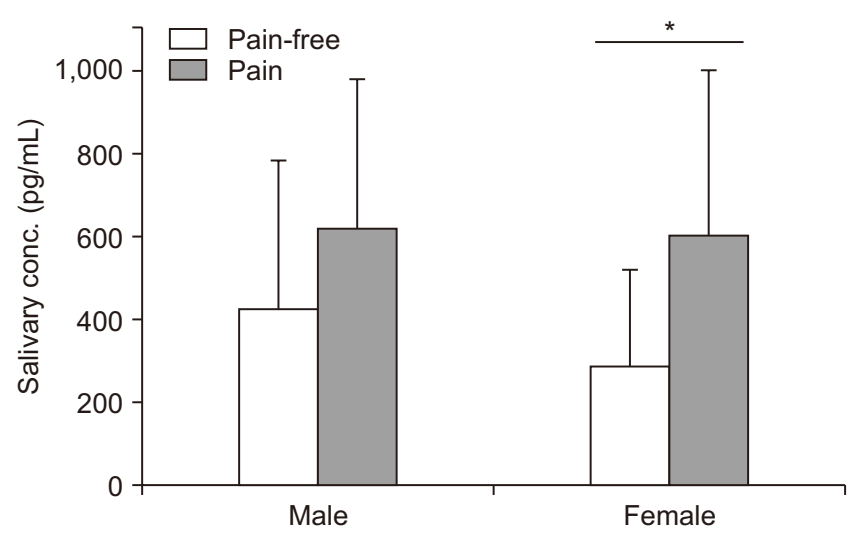

MMP-9

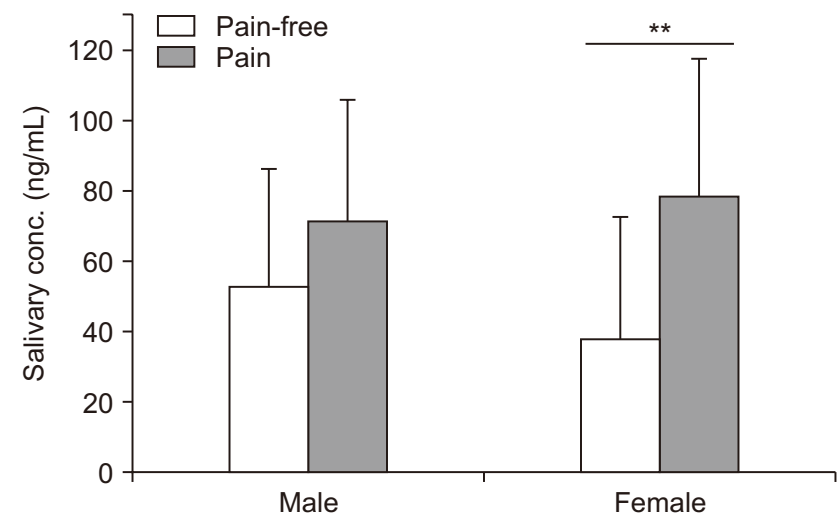

Fig. 6. Mean concentrations of IL-8 and MMP-9 according to sex of subjects. In female patients, the concentrations of salivary IL-8 and MMP9 were statistically higher in the pain groups than in the pain-free group (*** $p<0.05)$. But in men, there was no meaningful relationship between pain-free and pain group. The Mann - Whitney U-test was performed to compare concentrations of IL-8 and MMP-9 between groups. IL-8, interleukin-8; MMP-9, matrix metalloprotease 9.

and TMD accompanied by widespread palpation tenderness [22]. Jounger et al. [23] investigated patients with TMD myalgia, such as those participating in this study, and suggested that IL-8 was elevated in patients with jaw muscle pain during intramuscular microdialysis. The other studies reported that IL-8 could induce dose-dependent hyperalgesia and be related to pain signaling by directly activating primary nociceptive neurons [24,25]. Plasma levels of IL-8 were elevated by painful heat and cold stimuli and the maximum concentration of IL-8 was higher in older adults, who are more vulnerable to pain, than in younger adults [26]. The association between pain intensity and single nucleotide polymorphisms in $I L-8$ was investigated in patients with lung cancer $[27,28]$ and adenocarcinoma of the pancreas [29] and the polymorphism IL8-251T/A was confirmed to be an important predictor of severe pain in these patients.

MMPs, endopeptidases that can cleave the extracellular matrix, cytokines, and chemokines, are known to be involved in inflammation and to contribute to induction of neuropathic pain through inflammatory reactions and neural damage $[30,31]$. In comparison with healthy people, migraine patients suffering from severe pain had significantly greater plasma levels of MMP-9 [31,32]. Increased MMP9 levels in the periapical interstitial fluid was proportionate to the chance of pain on percussion in patients with apical periodontitis [33]. Dagher et al. [34] reported that MMP-9 could play a role as a biomarker in urinary chronic pelvic pain syndrome (UCPPS) and that the level of MMP-9 was significantly positively correlated with the pain severity in men with UCPPS.

In this study, the concentrations of IL-8 and MMP-9 were statistically higher in the saliva of patients with pain than in those without pain. The levels of IL-8 and MMP-9 were the highest values in the mild pain group (NRS 1-4) and the salivary levels in descending order were as follows: the moderate pain group (NRS 5-6), the severe pain group (NRS 7-10) and the control (NRS 0). Errors according to the various pain thresholds and personal perception may underlie this observation, or it may be that IL-8 and MMP-9 are truly expressed most strongly in the mildly painful stage. In another study involving myalgia patients with TMD, patients in the chronic pain group complained of more severe pain than those in the acute pain group [35]. Many previous studies have reported that chronic TMD patients exhibited more pain sensitivity to pressure pain, heat, and pinprick stimuli than TMD-free controls [36] and showed reduced pressure pain thresholds [37]. Since the intensity of pain that patients are feeling could be increased by changing to chronic TMD, it is estimated that IL-8 and MMP-9 levels are increased a little more specifically in acute pain, and thus, they could be decreased as pain becomes worse and old.

Acute and chronic pain involves different mechanisms of pain perception $[38,39]$. In cases of acute pain, the pain 
results from events that occur in response to local muscular injury; however, chronic pain is induced by abnormal responses in the central pain perception systems (e.g., central sensitization). There have been many reports that IL-8 and MMP-9 levels are high in patients with acute pain. The serum levels of IL-8 were significantly higher in patients with acute myofascial pain than in controls [40,41]. Compared to heathy individuals, the blood levels of IL-8 were markedly higher in patients with post-herpetic neuralgia [42]. MMP9 levels in serum were highly elevated in heathy men after high-intensity exercise that induced muscle pain [43] and in patients with acute appendicitis [44]. In this study, the salivary concentrations of IL-8 and MMP-9 were significantly higher in the pain group with a pain duration under 3 months than in the control group.

Female patients with TMD complained of pain much more frequently than male patients $[45,46]$. Extensive literature suggests that gender difference in pain results from estrogen levels, different mechanism of pain modulation, psychological factors, etc. [47,48]. In the high stress group, the number of women was about two times higher than that of men, and stress may have led to masticatory dysfunction [49]. Estrogen and inflammation could excite TMJ neuronal activity, which may lead to differential pain intensities between men and women [50]. In the present study, the levels of IL-8 and MMP-9 in saliva were significantly higher in female pain group than in controls, which indicated that the pain-induced IL-8 and MMP-9 upregulation could contribute more to pain perception or sensitivity in women than in men.

This study had several limitations. The sample number of patients was small because this study targeted TMD patients receiving no medication, while having no chronic diseases or inflammation, to exclude their effects on pain. The present study could have benefited from a greater sample size. The mean age of participants was below 30 years. Because the elderly have relatively more chronic pain conditions, the results of this study might be more meaningful for acute than for chronic pain. Finally, the salivary samples were collected only at the first visit of new patients. To compensate the individual differences of patients and draw more accurate conclusions, more studies are needed to assess the relationship between the levels of IL-8 and MMP-9 in patient saliva and pain change during the second visit.

In conclusion, pain could be one part of disease and the disease for itself. Object pain biomarkers could help provide suitable diagnosis, medical treatment and knowledge of the disease and recover patient's heath. To find easily and quickly usable salivary pain marker, the GEO database was searched, pain-related genes were analyzed and finally IL-8, MMP-9 were selected. This study showed a significant interrelationship between myalgia of TMD and the salivary concentrations of IL-8 and MMP-9. The differences were particularly much more in case of female and a pain duration under 3 months. This suggested the potential for IL-8 and MMP-9 to be used as salivary indicators of myalgia. Further studies are necessary to identify the sensitivity, specifity and odds ratio of IL-8 and MMP-9 as a biomarker of pain.

\section{CONFLICT OF INTEREST}

No potential conflict of interest relevant to this article was reported.

\section{ORCID}

Yang Mi Park

https://orcid.org/0000-0002-4508-2543

Yong-Woo Ahn

https://orcid.org/0000-0002-2197-0394

Sung-Hee Jeong

https://orcid.org/0000-0002-6296-4775

Hye-Min Ju

https://orcid.org/0000-0002-9252-6717

Hye-Mi Jeon

https://orcid.org/0000-0003-0007-5662

Kyung-Hee Kim

https://orcid.org/0000-0003-2922-6452

Soo-Min Ok

https://orcid.org/0000-0003-1776-371X

\section{REFERENCES}

1. Montes-Sandoval L. An analysis of the concept of pain. J Adv Nurs 1999;29:935-941. 
2. Shibata M, Kawai M, Matsukura T, Heike T, Okanoya K, MyowaYamakoshi M. Salivary biomarkers are not suitable for pain assessment in newborns. Early Hum Dev 2013;89:503-506.

3. Sobas EM, Reinoso R, Cuadrado-Asensio R, Fernández I, Maldonado MJ, Pastor JC. Reliability of potential pain biomarkers in the saliva of healthy subjects: inter-individual differences and intersession variability. PLoS One 2016;11:e0166976.

4. Von Korff M, Jensen MP, Karoly P. Assessing global pain severity by self-report in clinical and health services research. Spine (Phila Pa 1976) 2000;25:3140-3151.

5. Williamson A, Hoggart B. Pain: a review of three commonly used pain rating scales. J Clin Nurs 2005;14:798-804.

6. Hjermstad MJ, Fayers PM, Haugen DF, et al. Studies comparing Numerical Rating Scales, Verbal Rating Scales, and Visual Analogue Scales for assessment of pain intensity in adults: a systematic literature review. J Pain Symptom Manage 2011;41:10731093.

7. Conti PC, Costa YM, Gonçalves DA, Svensson P. Headaches and myofascial temporomandibular disorders: overlapping entities, separate managements? J Oral Rehabil 2016;43:702-715.

8. Cairns BE. Pathophysiology of TMD pain--basic mechanisms and their implications for pharmacotherapy. J Oral Rehabil 2010;37:391-410.

9. Javaid MA, Ahmed AS, Durand R, Tran SD. Saliva as a diagnostic tool for oral and systemic diseases. J Oral Biol Craniofac Res 2016;6:66-75.

10. Nunes LA, Mussavira S, Bindhu OS. Clinical and diagnostic utility of saliva as a non-invasive diagnostic fluid: a systematic review. Biochem Med (Zagreb) 2015;25:177-192.

11. Balan JJ, Rao RS, Premalatha BR, Patil S. Analysis of tumor marker CA 125 in saliva of normal and oral squamous cell carcinoma patients: a comparative study. J Contemp Dent Pract 2012;13:671-675.

12. Floriano PN, Christodoulides N, Miller CS, et al. Use of salivabased nano-biochip tests for acute myocardial infarction at the point of care: a feasibility study. Clin Chem 2009;55:1530-1538.

13. Smith SM, Dworkin RH, Turk DC, et al. The potential role of sensory testing, skin biopsy, and functional brain imaging as biomarkers in chronic pain clinical trials: IMMPACT considerations. J Pain 2017;18:757-777.

14. Jin EH, Zhang E, Ko Y, et al. Genome-wide expression profiling of complex regional pain syndrome. PLoS One 2013;8:e79435.

15. Treede RD, Rief W, Barke A, et al. Chronic pain as a symptom or a disease: the IASP Classification of Chronic Pain for the International Classification of Diseases (ICD-11). Pain 2019;160:19-27.

16. Shahzad A, Knapp M, Lang I, Köhler G. Interleukin 8 (IL-8)-a universal biomarker? Int Arch Med 2010;3:11.

17. Ahn SH, Cho YW, Ahn MW, Jang SH, Sohn YK, Kim HS. mRNA expression of cytokines and chemokines in herniated lumbar intervertebral discs. Spine (Phila Pa 1976) 2002;27:911-917.

18. Burke JG, Watson RW, McCormack D, Dowling FE, Walsh MG, Fitzpatrick JM. Intervertebral discs which cause low back pain secrete high levels of proinflammatory mediators. J Bone Joint Surg Br 2002;84:196-201.

19. Ang DC, Moore MN, Hilligoss J, Tabbey R. MCP-1 and IL-8 as pain biomarkers in fibromyalgia: a pilot study. Pain Med 2011;12:1154-1161.

20. Wang H, Moser M, Schiltenwolf M, Buchner M. Circulating cytokine levels compared to pain in patients with fibromyalgiaa prospective longitudinal study over 6 months. J Rheumatol 2008;35:1366-1370.

21. White AT, Light AR, Hughen RW, et al. Severity of symptom flare after moderate exercise is linked to cytokine activity in chronic fatigue syndrome. Psychophysiology 2010;47:615-624.

22. Slade GD, Conrad MS, Diatchenko L, et al. Cytokine biomarkers and chronic pain: association of genes, transcription, and circulating proteins with temporomandibular disorders and widespread palpation tenderness. Pain 2011;152:2802-2812.

23. Louca Jounger S, Christidis N, Svensson P, List T, Ernberg M. Increased levels of intramuscular cytokines in patients with jaw muscle pain. J Headache Pain 2017;18:30.

24. Ribeiro RA, Vale ML, Thomazzi SM, et al. Involvement of resident macrophages and mast cells in the writhing nociceptive response induced by zymosan and acetic acid in mice. Eur J Pharmacol 2000;387:111-118.

25. Oh SB, Tran PB, Gillard SE, Hurley RW, Hammond DL, Miller RJ. Chemokines and glycoprotein 120 produce pain hypersensitivity by directly exciting primary nociceptive neurons. J Neurosci 2001;21:5027-5035.

26. Cruz-Almeida Y, Aguirre M, Sorenson HL, Tighe P, Wallet SM, Riley JL 3rd. Age differences in cytokine expression under conditions of health using experimental pain models. Exp Gerontol 2015;72:150-156.

27. Reyes-Gibby CC, Wang J, Spitz M, Wu X, Yennurajalingam S, Shete S. Genetic variations in interleukin-8 and interleukin-10 are associated with pain, depressed mood, and fatigue in lung cancer patients. J Pain Symptom Manage 2013;46:161-172.

28. Reyes-Gibby CC, Spitz M, Wu X, et al. Cytokine genes and pain severity in lung cancer: exploring the influence of TNFalpha-308 G/A IL6-174G/C and IL8-251T/A. Cancer Epidemiol Biomarkers Prev 2007;16:2745-2751.

29. Reyes-Gibby CC, Shete S, Yennurajalingam S, et al. Genetic and nongenetic covariates of pain severity in patients with adenocarcinoma of the pancreas: assessing the influence of cytokine genes. J Pain Symptom Manage 2009;38:894-902.

30. Vafadari B, Salamian A, Kaczmarek L. MMP-9 in translation: from molecule to brain physiology, pathology, and therapy. J Neurochem 2016;139(Suppl 2):91-114.

31. Lakhan SE, Avramut M. Matrix metalloproteinases in neuropathic pain and migraine: friends, enemies, and therapeutic targets. Pain Res Treat 2012;2012:952906.

32. Imamura K, Takeshima T, Fusayasu E, Nakashima K. Increased plasma matrix metalloproteinase-9 levels in migraineurs. Headache 2008;48:135-139.

33. Martinho FC, Teixeira FF, Cardoso FG, et al. Clinical investigation of matrix metalloproteinases, tissue inhibitors of matrix metalloproteinases, and matrix metalloproteinase/tissue inhibitors of matrix metalloproteinase complexes and their networks in apical periodontitis. J Endod 2016;42:1082-1088.

34. Dagher A, Curatolo A, Sachdev M, et al. Identification of novel 
non-invasive biomarkers of urinary chronic pelvic pain syndrome: findings from the Multidisciplinary Approach to the Study of Chronic Pelvic Pain (MAPP) Research Network. BJU Int 2017;120:130-142.

35. Salmos-Brito JA, de Menezes RF, Teixeira CE, et al. Evaluation of low-level laser therapy in patients with acute and chronic temporomandibular disorders. Lasers Med Sci 2013;28:57-64.

36. Greenspan JD, Slade GD, Bair E, et al. Pain sensitivity risk factors for chronic TMD: descriptive data and empirically identified domains from the OPPERA case control study. J Pain 2011;12(11 Suppl):T61-T74.

37. Slade GD, Sanders AE, Ohrbach R, et al. Pressure pain thresholds fluctuate with, but do not usefully predict, the clinical course of painful temporomandibular disorder. Pain 2014;155:2134-2143.

38. Sessle BJ. The neural basis of temporomandibular joint and masticatory muscle pain. J Orofac Pain 1999;13:238-245.

39. Kimos P, Biggs C, Mah J, et al. Analgesic action of gabapentin on chronic pain in the masticatory muscles: a randomized controlled trial. Pain 2007;127:151-160.

40. Grosman-Rimon L, Kumbhare DA. Inflammatory biomarkers in acute myofascial pain. PM R 2016;8(9S):S155.

41. Grosman-Rimon L, Parkinson W, Upadhye S, et al. Circulating biomarkers in acute myofascial pain: a case-control study. Medicine (Baltimore) 2016;95:e4650.

42. Bäckryd E. Pain in the blood? Envisioning mechanism-based diagnoses and biomarkers in clinical pain medicine. Diagnostics (Basel) 2015;5:84-95.
43. van de Vyver M, Engelbrecht L, Smith C, Myburgh KH. Neutrophil and monocyte responses to downhill running: Intracellular contents of MP0, IL-6, IL-10, pstat3, and SOCS3. Scand J Med Sci Sports 2016;26:638-647.

44. Andersson M, Rubér M, Ekerfelt C, Hallgren HB, Olaison G, Andersson RE. Can new inflammatory markers improve the diagnosis of acute appendicitis? World J Surg 2014;38:2777-2783.

45. Ghurye S, McMillan R. Pain-related temporomandibular disorder - current perspectives and evidence-based management. Dent Update 2015;42:533-536, 539-542, 545-546.

46. Bueno CH, Pereira DD, Pattussi MP, Grossi PK, Grossi ML. Gender differences in temporomandibular disorders in adult populational studies: a systematic review and meta-analysis. J Oral Rehabil 2018;45:720-729.

47. Ribeiro-Dasilva MC, Fillingim RB, Wallet SM. Estrogen-induced monocytic response correlates with TMD pain: a case control study. J Dent Res 2017;96:285-291.

48. Abrahamsen R, Zachariae R, Svensson P. Effect of hypnosis on oral function and psychological factors in temporomandibular disorders patients. J Oral Rehabil 2009;36:556-570.

49. Anna S, Joanna K, Teresa S, Maria G, Aneta W. The influence of emotional state on the masticatory muscles function in the group of young healthy adults. Biomed Res Int 2015;2015:174013.

50. Flake NM, Bonebreak DB, Gold MS. Estrogen and inflammation increase the excitability of rat temporomandibular joint afferent neurons. J Neurophysiol 2005;93:1585-1597. 